\title{
REQUERIMIENTO DE POTASIO DE UN HÍBRIDO Y UN CULTIVAR DE CANOLA (BRASSICA NAPUS CV. OLEÍFERA) Y SU EFECTO EN LA PRODUCCIÓN EN DOS SUELOS ANDISOLES DE LA REGIÓN DE LA ARAUCANÍA
}

\author{
POTASSIUM REQUIREMENT OF A HYBRID AND CULTIVATE CANOLA \\ (BRASSICA NAPUS CV. OLEÍFERA) AND ITS EFFECT ON PRODUCTION IN \\ TWO SOIL OF THE ANDISOLS REGION OF ARAUCANÍA
}

\author{
Hernán Pinilla Quezada1 ; Claudia Bravo Bustos²; Héctor Sanhueza Roa ${ }^{3}$
}

\begin{abstract}
RESUMEN
Se realizó un ensayo con el propósito de evaluar el requerimiento de potasio de un híbrido y un cultivar de canola (Brassica napus cv. Oleífera) y su efecto en la producción y contenido de materia grasa en dos suelos andisoles de la región de la Araucanía. Se utilizó un diseño de bloques al azar, con cuatro dosis, $0,50,100,150 \mathrm{~kg} \mathrm{de} \mathrm{K}_{2} \mathrm{O}$, y cuatro repeticiones. Las variables a evaluar fueron: rendimiento del grano, contenido de materia grasa y requerimiento de potasio por quintal. Los resultados indican que las dosis de potasio utilizadas no afectaron de forma significativa el rendimiento de los cultivares utilizados ni el contenido de materia grasa en ambos suelos. El requerimiento promedio de potasio fue de 2,7 y 2,6 kg de $\mathrm{K}$ por qqm para Artus y Tequila, respectivamente.
\end{abstract}

Palabras clave: Fertilización potásica, Brassica napus cv Oleífera, materia grasa raps.

\section{ABSTRACT}

A study was carried out to assess the requirement of potassium and a hybrid cultivar of canola (Brassica napus $c v$. Oleifera) and its effect on production and fat content on two soil andisols in the region of Araucania. We used a randomized block design with four doses, 0, 50, 100, $150 \mathrm{~kg}$ of $\mathrm{K}_{2} \mathrm{O}$ and four repetitions. The variables assessed were: grain yield, fat content and requirement of potassium per quintal. The results indicate that the doses of potassium used, did not significantly affect the performance of the cultivars used, or the fat content in both soils. The average requirement of potassium was 2.7 and $2.6 \mathrm{~kg}$ of $\mathrm{K}$ qqm for Artus and Tequila, respectively.

Key words: K fertilization, Brassica napus cv Oleífera, fat raps.

\section{INTRODUCCIÓN}

El raps es una oleaginosa importante a nivel mundial y nacional, de fácil adaptación en áreas con estaciones largas y con bajas temperaturas, lo que le ha permitido establecerse en el sur de Chile.

Su producción se ha destinado a la elaboración de alimentos para animales, especialmente salmones y aves, y en una menor proporción al consumo humano. Adicionalmente, se ha utilizado como materia prima en la elaboración de biodiésel.
Los costos de producción del raps han afectado la decisión de sembrar esta oleaginosa, por el alto valor de los insumos, entre ellos el potasio, nutriente que junto al nitrógeno son los de más alta exigencia para el cultivo.

A pesar de que el requerimiento total de $\mathrm{K}$ del cultivo es muy alto, la extracción en la semilla es de un 15\% aproximadamente, permaneciendo el resto en los residuos de cosecha (Orlovius, 2006).

De acuerdo a lo señalado por Bullock (1990) en estudios realizados en Canadá y Europa el

\footnotetext{
1 Ingeniero Agrónomo, Magíster en Fertilidad de Suelos, Académico Investigador FCAF. Universidad de La Frontera. Temuco, Chile. E-mail: hpin@ufro.cl

2 Ingeniero Agrónomo. E-mail: cbrav015mail.com

3 Ingeniero Ejecución Agrícola. Académico FCAF, Universidad de La Frontera. Temuco, Chile. E-mail: hsanhue@ufro.cl
} 
requerimiento de $\mathrm{K}$ varía entre 3,2 a $4 \mathrm{~kg}$ de K/qqm, respectivamente. Pinochet (1988) indica en estudios realizados en el país que el requerimiento de este nutriente es de 3,6 kilos de K/qqm. Merrien et al. (1988) señalan, en estudios realizados en Francia, requerimiento de 10 kilos de K/qqm.

Zaman et al. (2004) encontraron incrementos en el número de vainas y granos por vaina al aplicar $125 \mathrm{~kg}$ de $\mathrm{K}_{2} \mathrm{O} / \mathrm{ha}$ en un suelo con una disponibilidad inicial de $131 \mathrm{mg} / \mathrm{kg}$. Carmody y Cox (2001) y Brennan y Bolland (2007b) obtuvieron respuestas a aplicaciones de potasio en el rendimiento al aplicar este nutriente en dosis de 25 a $30 \mathrm{~kg} / \mathrm{ha}$ en suelos arenosos con niveles menores a $85 \mathrm{mg} / \mathrm{kg}$ de $\mathrm{K}$ intercambiable.

Estudios efectuados por Thomas (2007) han establecido que el nivel crítico de este nutriente en el suelo es de $110 \mathrm{mg} / \mathrm{kg}$. Brennan y Bolland (2007b) señalan que valores menores a $60 \mathrm{mg} / \mathrm{kg}$ de $\mathrm{K}$ intercambiable son insuficientes para la producción del raps canola.

Considerando la variabilidad señalada en la literatura respecto al requerimiento de $\mathrm{K}$ del raps canola y el nivel crítico de este nutriente en el suelo, el objetivo de la presente investigación fue establecer el requerimiento de $\mathrm{K}$ de un híbrido y un cultivar de canola (Brassica napus cv. Oleífera) y su efecto en la producción y contenido de materia grasa en un suelo andisol de la región de La Araucanía, con diferente disponibilidad inicial de este nutriente.

\section{MATERIALES Y MÉTODOS}

El ensayo se realizó durante la temporada agrícola 2006-2007 en un suelo andisol de lomaje de la serie Gorbea y en suelo andisol plano de la serie Freire. El análisis químico de suelos se presenta en el Cuadro 1.

En el ensayo se utilizó un diseño factorial de $2 \times 4$, en arreglos de bloques al azar, con el híbrido Artus y el cultivar Tequila: cuatro dosis de potasio $\left(0,50,100,150 \mathrm{~kg} \mathrm{~K}_{2} \mathrm{O} / \mathrm{ha}\right)$ y cuatro repeticiones por cada uno de los tratamientos. El tamaño de las parcelas fue de $12 \mathrm{~m}^{2}$, con un total de 64 parcelas experimentales.

La especie utilizada fue Brassica napus cv. Oleífera, tipo canola; el ensayo se estableció 30 de abril de 2006 en el trumao plano y el 6 de mayo en el trumao de lomaje.

La fertilización base se realizó tomando como referencia el suelo con mayores limitaciones,
Cuadro 1

Características químicas de los suelos utilizados

\begin{tabular}{|l|c|c|}
\hline \multirow{2}{*}{\multicolumn{1}{|c|}{ Componente }} & \multicolumn{2}{c|}{ Contenido } \\
\cline { 2 - 3 } & Trumao plano & Trumao lomaje \\
\hline Fósforo Olsen $(\mathrm{mg} / \mathrm{kg})$ & 10 & 16 \\
\hline Potasio $(\mathrm{mg} / \mathrm{kg})$ & 98 & 149 \\
\hline $\mathrm{pH}(\mathrm{en}$ agua) & 5,65 & 5,32 \\
\hline Calcio (cmol+/kg) & 4,22 & 2,26 \\
\hline Magnesio (cmol+/kg) & 0,61 & 0,27 \\
\hline Sodio (cmol+/kg) & 0,13 & 0,03 \\
\hline Aluminio (cmol+/kg) & 0,09 & 0,16 \\
\hline Suma bases (cmol+/kg) & 5,21 & 2,97 \\
\hline S (mg/kg) & 9 & 27 \\
\hline Sat. aluminio (\%) & 1,7 & 5,11 \\
\hline
\end{tabular}

Fuente; Laboratorio de Análisis Químico de Suelos y Plantas. Instituto de Agroindustrias, Universidad de La Frontera.

utilizando la metodología propuesta por Rodríguez et al. (2001). A la siembra se aplicaron $30 \mathrm{~kg}$ de $\mathrm{N} ; 200 \mathrm{~kg}$ de $\mathrm{P}_{2} \mathrm{O}_{5}: 54 \mathrm{~kg}$ de $\mathrm{CaO} ; 38 \mathrm{~kg}$ de $\mathrm{MgO}$; $26 \mathrm{~kg}$ de $\mathrm{S} ; 2 \mathrm{~kg}$ de B y $3 \mathrm{~kg}$ de $\mathrm{Zn}$. La fertilización se completó con 69 unidades de nitrógeno aplicadas al estado 14 a 15 y 103 unidades de nitrógeno aplicadas en el estado 30, completando 200 unidades de nitrógeno por hectárea.

El control químico de malezas se realizó con Acetamida en dosis de 2,5 lt/ha y Tepraloxydim en dosis de 0,75 lt/ha, al estado 13 a 14, más Picloran en dosis de $120 \mathrm{cc} /$ ha y Clopiralid en dosis de 250 cc/ha, al estado de 14 a 15 .

En el control de plagas y enfermedades se utilizó Lambdacyhalotrina en dosis de $150 \mathrm{gr} / \mathrm{ha}$ en el estado 10 y 30 , Flusilazole en dosis de $100 \mathrm{cc} / \mathrm{h}$ a al estado de 14 a 15, Pirimicarb en dosis de 300 gr/ha en el estado 65, y se aplicó Boscalid + Pyraclostrobin en dosis de $250 \mathrm{cc} / \mathrm{ha}$ al estado 57 y 60 .

Las evaluaciones realizadas fueron rendimiento del grano, contenido de materia grasa, y requerimiento de potasio, por quintal de grano.

Para determinar los efectos de las dosis de potasio en el híbrido y el cultivar los resultados fueron sometidos a una prueba de análisis de varianza, y los promedios fueron comparados mediante la prueba de rango múltiple de Tukey con un nivel de significancia de $95 \%$, utilizando el programa computacional SPSS. 


\section{PRESENTACIÓN Y DISCUSIÓN DE RESULTADOS}

\section{RENDIMIENTO DEL GRANO}

En la Figura 1 se presenta el rendimiento de grano obtenido en ambas localidades para el híbrido y el cultivar Tequila. No se presentaron diferencias significativas en los rendimientos entre el cultivar y el híbrido en los suelos utilizados.

Estos resultados concuerdan con lo señalado por Brennan y Bolland (2007b), quienes no encontraron diferencias significativas en la producción de grano al utilizar diferentes cultivares e híbridos de canola.

Por otro lado, Shi et al. (2004) sostienen que los híbridos tienen un mayor potencial productivo, a diferencia de los cultivares tradicionales, que tienen un menor potencial de rendimiento y una mayor adaptación a suelos deficientes en $\mathrm{K}$, lo cual no concuerda con el híbrido y cultivar utilizados en este estudio, donde ambos tuvieron rendimientos similares.

Respecto a las dosis de potasio utilizadas, no se presentaron diferencias significativas en ninguno de los suelos utilizados. Los resultados del presente estudio son coincidentes con los presentados por Govahi y Saffari (2006), quienes han señalado que incrementos en la aplicación de K no se traducen en un aumento en la producción de semillas.

Estos resultados estarían confirmando lo indicado por Zaman et al. (2004) quienes evaluaron en un suelo con $131 \mathrm{mg} / \mathrm{kg}$ de potasio intercambiable diferentes dosis de este nutriente, sin obtener respuesta a aplicaciones crecientes de $\mathrm{K}$ que alcanzaron a $150 \mathrm{~kg}$ de $\mathrm{K}_{2} \mathrm{O} / \mathrm{ha}$. En el presente estudio, con un nivel de potasio intercambiable de 98 y $149 \mathrm{mg} /$ $\mathrm{kg}$ y con dosis máxima de $150 \mathrm{~kg}$ de $\mathrm{K}_{2} \mathrm{O}$ no hubo

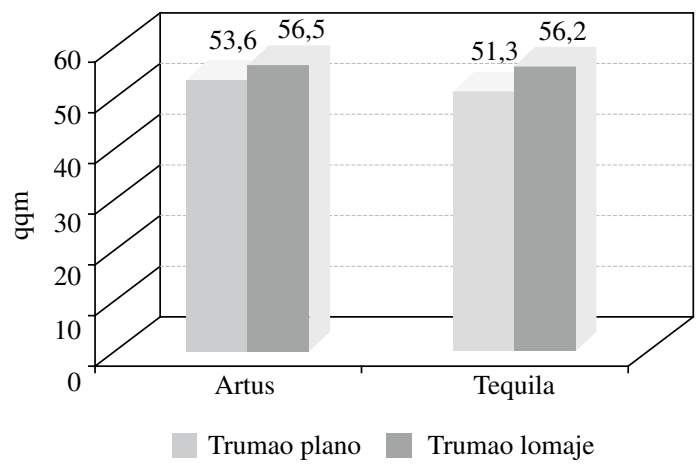

Figura 1. Rendimiento de grano en Artus y Tequila, en dos andisoles de la IX Región, expresado en qqm/ha. diferencias en el rendimiento con las distintas dosis de $\mathrm{K}$ evaluadas.

Terry et al. (2007) señalan que si la disponibilidad de este nutriente es entre $81-160 \mathrm{mg} / \mathrm{kg}$ se debería aplicar K para incrementar los rendimientos. En este experimento no se produjeron respuestas significativas en rendimiento por efecto de las dosis de potasio, lo que estaría indicando que el nivel de potasio presente en ambos suelos es suficiente para satisfacer los requerimientos de $52 \mathrm{qqm} / \mathrm{ha}$, aproximadamente.

\section{MATERIA GRASA}

En las Figuras 3 y 4 se presenta el porcentaje de materia grasa presente en las semillas de canola de acuerdo a los cultivares y dosis de potasio aplicadas en ambos suelos.

Los resultados obtenidos indican que hubo diferencias en el contenido de materia grasa entre los cultivares, con valores promedio de 49,9\% para Artus y de $53,1 \%$ para Tequila. El incremento en el contenido de materia grasa representa una

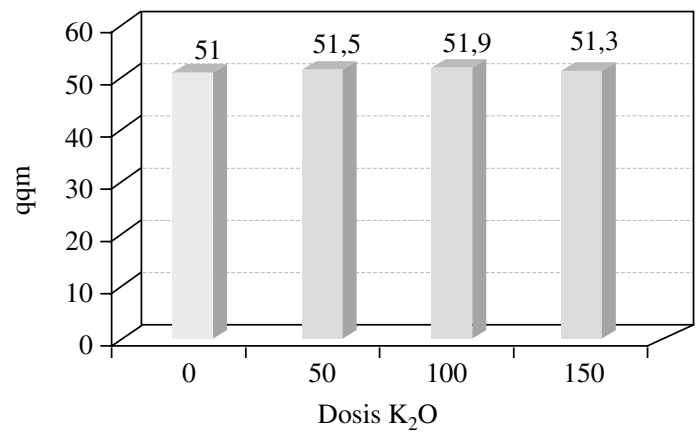

Figura 2. Efecto de las dosis de potasio en el rendimiento de grano, expresado en qqm/ha.

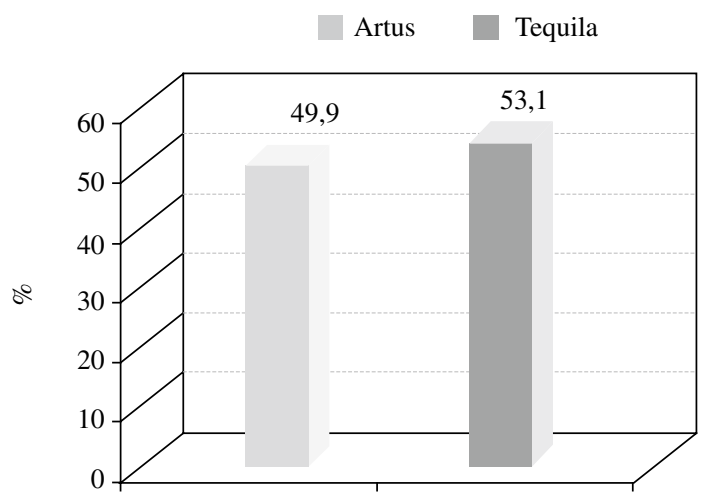

Figura 3. Contenido de materia grasa en Artus y Tequila, expresado en porcentaje. 


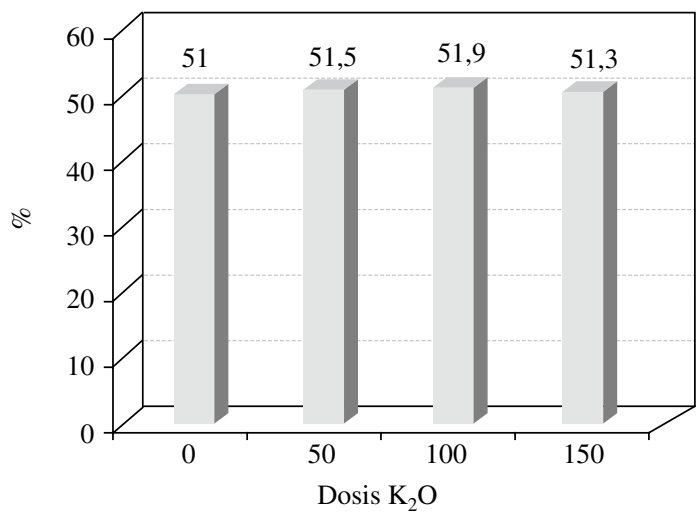

Figura 4. Efecto de las dosis de potasio en el contenido de materia grasa, expresado en porcentaje.

ventaja económica, debido a que la norma de calidad para la comercialización de raps bonifica su precio, con contenidos sobre $50 \%$ de materia grasa. Estas diferencias en el contenido de materia grasa son atribuibles a la genética de los cultivares evaluados.

Respecto a las dosis de potasio no se produjeron incrementos relevantes en el contenido de materia grasa en los suelos utilizados, obteniéndose concentraciones medias de $51,0 \%$ para el testigo y de $51,3 \%$ al aplicar $150 \mathrm{~kg} \mathrm{de} \mathrm{K}_{2} \mathrm{O} / \mathrm{ha}$. Estos resultados concuerdan con lo descrito por Govahi y Saffari, 2006; Brennan y Bolland, 2006; Brennan y Bolland, $2007 \mathrm{~b}$, quienes señalaron que el potasio no afecta el contenido de materia grasa en el grano.
Los resultados del presente estudio difieren de lo indicado por Zaman et al. (2004) quienes afirman que al aumentar la dosis de potasio disminuye el contenido de materia grasa en las semillas de canola, presentándose el mayor valor cuando no se aplica potasio.

\section{REQUERIMIENTO DE POTASIO}

En los Cuadros 2 y 3 se indica la absorción total de potasio por efecto de los diferentes cultivares y las dosis utilizadas.

De acuerdo a los resultados presentados se puede señalar que la absorción de potasio para los dos cultivares, y en ambos suelos, fue similar, concordando con lo señalado por Brennan y Bolland (2007 a), quienes indicaron que los diferentes cultivares utilizados en su estudio no tuvieron diferencias importantes en la absorción de potasio.

Según los resultados de los Cuadro 2 y 3 , y los rendimientos obtenidos por cada cultivar, los requerimientos promedios de $\mathrm{K}$ por quintal de grano producido serían 2,7 y 2,6 kg de K/qqm para Artus y Tequila, respectivamente. Estos requerimientos de K/qqm son menores a los obtenidos por Pinochet (1988), Bullock (1990) de 3,6; y 3,2 a 4,0 kg de K por quintal, y claramente diferentes a los citados por Merrien et al. (1988) y Orlovius (2006) de $10 \mathrm{~kg}$ de potasio por quintal de grano producido.

\section{Cuadro 2}

Potasio absorbido en la semilla, residuos y absorción total de $\mathrm{K}$ en Artus y Tequila con diferentes dosis de $\mathrm{K}$ utilizadas, en un trumao plano con $98 \mathrm{mg} / \mathrm{kg}$ de $\mathrm{Ki}$

\begin{tabular}{|c|c|c|c|c|c|}
\hline \multirow[b]{2}{*}{ Dosis de $\mathrm{K}_{2} \mathrm{O}$} & \multicolumn{5}{|c|}{ Artus } \\
\hline & $\begin{array}{c}\% \mathrm{~K} \\
\text { Semilla }\end{array}$ & K kg/ha Semilla & \% K Residuo & K kg/ha Residuo & Absorción kg K/ha \\
\hline 0 & 0,45 & 22 & 0,99 & 91 & 113 \\
\hline 50 & 0,46 & 22 & 1,24 & 108 & 130 \\
\hline 100 & 0,50 & 24 & 1,36 & 120 & 144 \\
\hline \multirow[t]{2}{*}{150} & 0,45 & 22 & 1,3 & 118 & 140 \\
\hline & \multicolumn{5}{|c|}{ Tequila } \\
\hline 0 & 0,46 & 21 & 1,01 & 93 & 114 \\
\hline 50 & 0,49 & 23 & 1,17 & 110 & 132 \\
\hline 100 & 0,52 & 24 & 1,28 & 119 & 143 \\
\hline 150 & 0,52 & 25 & 1,36 & 130 & 155 \\
\hline
\end{tabular}




\section{Cuadro 3}

Potasio absorbido en la semilla, residuos, y absorción total de K, en Artus y Tequila con diferentes dosis de K utiliza $\neq$ das, en un trumao de lomaje, con 149 mg/kg de Ki

\begin{tabular}{|l|c|c|c|c|c|}
\hline \multirow{2}{*}{ Dosis de K2O } & \multicolumn{5}{|c|}{ Artus } \\
\cline { 2 - 6 } & \% K Semilla & K kg/ha Semilla & \% K Residuo & K kg/ha Residuo & Absorción kg K/ha \\
\hline 0 & 0,47 & 23 & 0,93 & 86 & 110 \\
\hline 50 & 0,53 & 26 & 1,15 & 107 & 133 \\
\hline 100 & 0,51 & 25 & 1,24 & 113 & 138 \\
\hline 150 & 0,52 & 28 & 1,03 & 104 & 132 \\
\hline & & & Tequila & 87 & 109 \\
\hline 0 & 0,44 & 21 & 0,89 & 107 & 130 \\
\hline 50 & 0,45 & 23 & 1,02 & 107 & 131 \\
\hline 100 & 0,47 & 24 & 1,03 & 109 & 132 \\
\hline 150 & 0,45 & 23 & 1,04 & & \\
\hline
\end{tabular}

En el presente estudio y en ambos cultivares la distribución del $\mathrm{K}$ a la cosecha fue de $18 \%$ de potasio en la semilla y de $82 \%$ en los residuos. Estos resultados son similares a los obtenidos por Orlovius (2006) quien señala que la acumulación de potasio en las semillas es de un $15 \%$ y que en los residuos se concentra un $85 \%$ del total del potasio absorbido por el cultivo.

La concentración promedio de potasio en la semilla en ambos cultivares fue de $0,48 \%$, valores similares a los obtenidos por Brennan y Bolland (2006), Terry et al. (2007), quienes señalan que las concentraciones de $\mathrm{K}$ en el grano se distribuyen entre un rango de $0,55 \%$ a $0,73 \%$.

La concentración de potasio en los residuos fue similar para ambos cultivares, con un valor promedio de $1,13 \%$, lo que difiere de lo señalado por Orlovius (2006) quien obtuvo concentraciones de potasio de un 3,37\% en la etapa de maduración.

A medida que aumentan las dosis de potasio aplicadas se incrementó levemente la absorción total de potasio en Artus y Tequila, debido a que el cultivo tiende a aumentar la absorción por la mayor disponibilidad de este nutriente en el suelo. Sin embargo, este aumento no provocó incrementos significativos en el rendimiento. La absorción total de $\mathrm{K}$ de $130 \mathrm{~kg}$ de $\mathrm{K} / \mathrm{ha}$ es muy inferior a los 400 kilos de K absorbidos citado por Orlovius, (2006). $\mathrm{El}$ hecho de que los rendimientos promedios en el presente estudio fueron de $52 \mathrm{qqm} / \mathrm{ha}$ estaría indicando que la absorción de potasio es adecuada y que probablemente en los estudios de Orlovius (2006) se haya producido un consumo de lujo de este nutriente.

\section{CONCLUSIONES}

- No se produjeron, en ninguno de los suelos, diferencias significativas de rendimiento de grano entre el híbrido y el cultivar.

- Aplicaciones de potasio no produjeron, en los suelos estudiados, diferencias significativas de rendimiento.

- Los contenidos medios de materia grasa en ambos suelos fueron superiores en el cultivar Tequila y las dosis crecientes de potasio no afectaron los contenidos promedios de materia grasa de los cultivares.

- $\quad$ El requerimiento promedio de $\mathrm{K}$ fue de $2,7 \mathrm{~kg}$ de K/qqm y de 2,6 kg de K/qqm para Artus y Tequila respectivamente. 


\section{LITERATURA CITADA}

BRENNAN, R.; BOLLAND, M. 2006. Soil and tissue tests to predict the potassium requirements of canola in southwestern Australia. Australian Journal of Experimental Agriculture. Australia. 46: 675-679.

BRENNAN, R.; BOLLAND, M. 2007a. Comparing the potassium requirements of canola and wheat. Australian Journal of Agricultural Research. Australia. 58: 359366.

BRENNAN, R.; BOLLAND, M. 2007b. Influence of potassium and nitrogen fertiliser on yield, oil and protein concentration of canola (Brassica napus L) grain harvested in southwestern Australia. Australian Journal of Experimental Agriculture. Australia. 47: 976-983.

BULLOCK, D. 1990. Canola Fertility. Illinois Fertilizer Conference Proceedings January. 23-24, 1990. 12 de enero del 2008, http://frec.cropsci.uiuc.edu/1990/report9/.

CARMODY, P.; COX, A. 2001. Profitable canola production in the Northern grainbelt Of western Australia. Boletin 4491. Segunda edición. Agriculture Western Australia.

GOVAHI, M.; SAFFARI, M. 2006. Effect of potassium and sulphur fertilizers on yield, yield components and seed quality of spring canola (Brassica napus L.) seed. Journal of Agronomy. Kerman Iran. 5 (4): 577-582.

MERRIEN, A.; PAlleaU, J.P.; MAISSONNEUVE, C. 1988. Besoins en elements minéraux du colza cultivé en
France. In: Physiologie et élaboration du rendement du colza dhiver, CETIOM, F-75116 Paris.

ORLOVIUS, K. 2006. K. Fertilizing for High Yield and Quality Oilseed Rape. IPI Boletín No 16. Alemania, p. 125.

PINOCHET, D. 1988. Nutrición y fertilización del raps. En: Fuentealba. Producción de raps Oleaginoso. Instituto de Producción y Sanidad Vegetal. Universidad Austral. Valdivia, Chile. Cap. 2: 59-90.

RODRÍGUEZ, P.; PINOCHET, D.; MATUS, F. 2001. Fertilización de los cultivos. LOM Ediciones Santiago. Chile, 117 pp.

SHI, W.; WANG, X.; YAN, W. 2004. Distribution patterns of available $\mathrm{P}$ and $\mathrm{K}$ in rape rhizosphere in relation to genotypic difference. Plant and Soil (China). 216: 11-16.

TERRY, J.; RENGEL, Z.; QIFU, M.; BORDEN, J. 2007. Differential accumulation patterns of phosphorus and potassium by canola cultivars compared to wheat. J. Plant Nutr. Soil Sci. Australia.170: 404-411.

THOMAS, P. 2007. Canola Response to Potassium Fertilizer. Chapter 9. Soil Fertility. 12 de enero del 2008, http://www. canola-council.org/chapter9.aspx\#ch9_sec12b

ZAMAN, H.; ASGHAR MALIK, M.; FARRUKH, M.; AZIZ, I. 2004. Effect of Different Potassium Fertilization Levels on Growth, Seed Yield and Oil Contents of Canola (Brassica napus L.). International Journal of Agriculture \& Biology. Pakistan. 6: 557-559. 